\title{
Probing the dynamical structure of $\delta$ Cephei atmosphere
}

\author{
N. Nardetto ${ }^{1}$, A. Fokin ${ }^{1,2}$, D. Mourard ${ }^{1}$, and Ph. Mathias ${ }^{1}$ \\ 1 Observatoire de la Côte d'Azur, Dpt. Gemini, UMR 6203, 06130 Grasse, France \\ e-mail: Nicolas.Nardetto@obs-azur.fr \\ 2 Institute of Astronomy of the Russian Academy of Sciences, 48 Pjatnitskaya Str., Moscow 109017, Russia \\ Received 29 September 2005 / Accepted 11 March 2006
}

\begin{abstract}
Context. Limb darkening and the projection factor are currently two limiting aspects of the interferometric Baade-Wesselink method of Cepheid distance determination.

Aims. We first quantify the impact of the phase dependence of limb darkening on the derived distance. We then study a new way to probe the dynamical structure of Cepheid's atmosphere through spectro-interferometric observations.

Methods. A hydrodynamical model of $\delta \mathrm{Cep}$ is used to derive stellar disk intensity distribution in the continuum and in different spectral lines, together with the corresponding wavelength- and phase-dependent visibility curves.

Results. We find that considering a constant limb darkening in the visible leads to a systematic shift of about 0.02 in phase on the angular diameter curve. The derived distance is, however, not affected by this effect. Otherwise, for a spectroscopic resolution of $R=12000$ in the visible, we find in the most favourable case (maximum contraction velocity) a signature on the visibility curve of about $7 \%$ that is clearly detectable by current spectro-interferometers. Nevertheless, the projection factor has only a $1 \%$ (or less) effect on the visibility curve.

Conclusions. The spectro-interferometry provides a new geometric view of Cepheid's atmosphere. However, the combination of different techniques (high resolution spectroscopy, spectro- and differential-interferometry) are now needed to efficiently constrain the physical parameters of Cepheids' atmosphere and, in particular the projection factor.
\end{abstract}

Key words. techniques: interferometric - stars: atmospheres - stars: distances - stars: oscillations - stars: variables: Cepheids

\section{Introduction}

Long-baseline interferometers currently provide a new quasigeometrical way to calibrate the Cepheid period-luminosity relation. Indeed it is now possible to determine the distance of galactic Cepheids up to $1 \mathrm{kpc}$ with the interferometric BaadeWesselink method, hereafter IBW method (Kervella et al. 2004); see also Sasselov \& Karovska (1994).

However, the limb darkening and the projection factor remain two limiting aspects of the IBW method. Interferometric measurements lead to angular diameter estimations over the whole pulsation period, while the stellar radius variations can be deduced from the integration of the pulsation velocity curve deduced from line profiles by the so-called projection factor $p$. Therefore, in order to provide a correct estimate of the distance, the angular and the linear estimations of the Cepheid diameter used in the IBW method have to correspond to the same layer.

In a previous paper (Nardetto et al. 2004, hereafter Paper I), we quantified the average value of the projection factor in different cases: spectroscopy, spectral-line, and wide-band interferometry. The conclusion is that a bias of $6 \%$ is possible on the derived distance if an incorrect projection factor is used. This theoretical result has been confirmed using the CHARA interferometer by Mérand et al. (2005).

Limb darkening is another important parameter in the IBW method. It must be known correctly to estimate the angular diameter of the star. In addition, its phase-dependence is of crucial importance in the context of the last generation of long-baseline interferometers. An important study of the wavelength- and phase-dependence of the limb-darkening, based on a hydrodynamical model, has been performed by Marengo et al. $(2002,2003)$. As expected they conclude that limbdarkening variation is larger in the visible (about 1\%) than in the infrared (about $0.2 \%$ ).

Based on the $\delta$ Cep model of Paper I, we first study the impact of the phase-dependence of the limb-darkening on the derived distance through stellar-disk intensity distribution in the continuum (Sect. 2). In Sect. 3, we present intensity distributions corresponding to four spectral lines formed at different depths in the atmosphere of the star. We then derive wavelength- and phase-dependence of the visibility in order to guide a new geometrical study of the projection factor.

\section{The effect of constant limb-darkening on distance determination}

We apply the same full-amplitude hydrodynamical model for $\delta$ Cep that we used in Paper I. Stellar-disk intensity distributions in the continuum (in the vicinity of the Fe I 6003.012 $\AA$ line) are used to study the limb-darkening. The photospheric pulsation velocity is represented in Fig. 1 as a function of the phase, together with the corresponding intensity distributions. These distributions are represented as a function of $\mu=\cos \theta$, where $\theta$ is the angle between the line of sight and the normal to the star. Intensity distributions are close to zero at the extreme limb of the disk, according to the atmosphere extension: $\frac{I(\mu)}{I(1)}<0.01$ for $\mu<0.2$ at the maximum radius phase $(\phi=0.39)$, and for $\mu<0.08$ at the minimum radius phase $(\phi=0.89)$. 


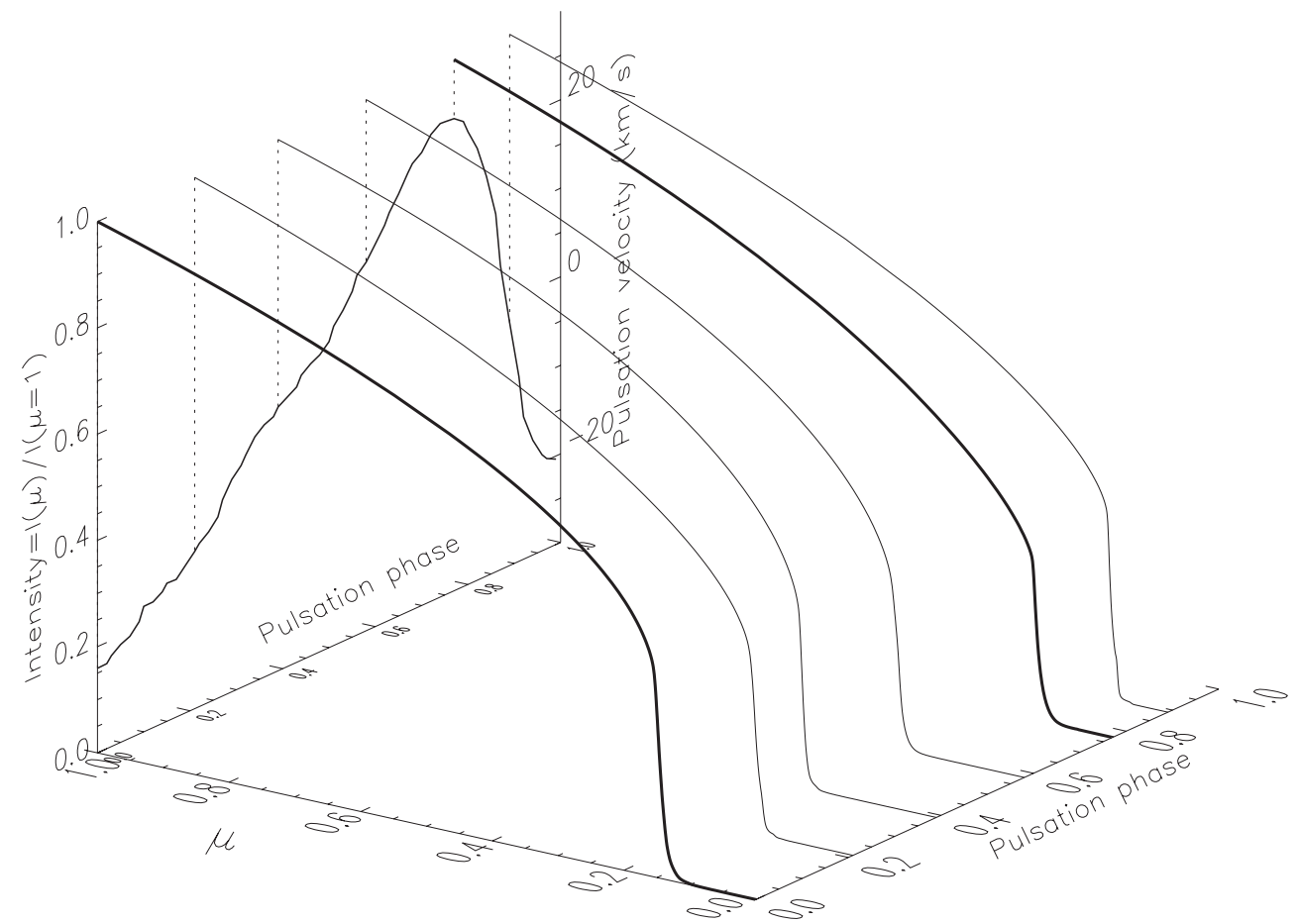

Fig. 1. A 3D diagram that represents the normalized stellar disk intensity distribution in the continuum as a function of $\mu$ (see text) for different phases. The vertical plot (arbitrary unit) represents the photospheric pulsation velocity as a function of the phase (in the heliocentric frame). Two distributions (in accentuated) are interesting because they correspond to extreme cases in limb-darkening.

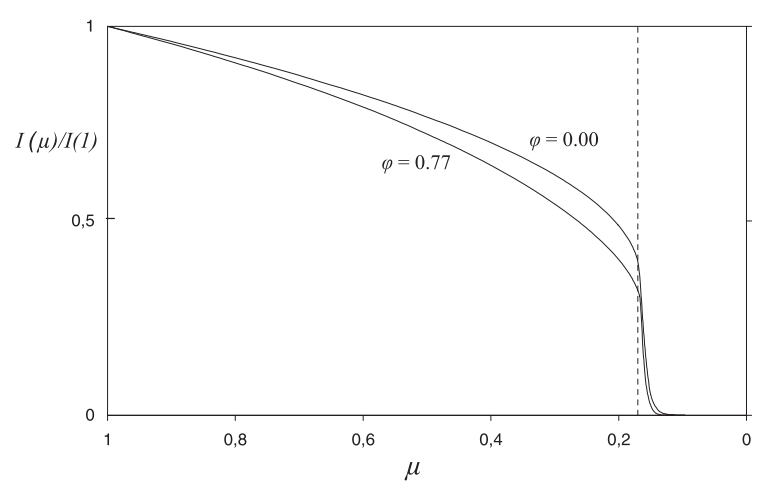

Fig. 2. Details of the two intensity distributions for phases 0.77 and 0.00 as a function of $\mu$. The profile corresponding to the highest velocity at contraction $(\phi=0.77)$ is the most limb-darkened, while the profile corresponding to the highest velocity at expansion $(\phi=0.0)$ is the lowest limb-darkened. The vertical dashed line represents the photosphere of the star defined by $\tau_{\mathrm{c}}=2 / 3$. The photosphere corresponds to the same value of $\mu$ for the two pulsation phases considered here.

The two intensity distributions are interesting because they correspond to extreme cases of pulsation velocity. The profile corresponding to the highest velocity at contraction $(\phi=0.77)$ is the most limb-darkened, while the profile corresponding to the highest velocity at expansion $(\phi=0.0)$ is the least limbdarkened. They are represented in Fig. 2 as a function of $\mu$. We also indicate the photosphere of the star defined by $\tau_{c}=2 / 3$, where $\tau_{\mathrm{c}}$ is the optical depth in the continuum.

We now quantify these qualitative results through an estimation of the $k$-parameter in the visible continuum defined as

$k(\phi)=\frac{\theta_{\mathrm{UD}}(\phi)}{\theta_{\text {photo }}(\phi)}$ where $\theta_{\text {photo }}$ is the photospheric angular diameter derived directly from the model and $\theta_{\mathrm{UD}}$ the angular diameter of the uniform disk. By definition, the uniform disk-angular diameter does not include the center-to-limb variation of the modeled intensity distribution, but in turn, it is sensitive to the limb darkening variation with the pulsation phase. The $k$-parameter is thus indirectly linked to the limb-darkening of the star. We can separate the average value of the $k$-factor and its phase-dependence: $k(\phi)=k_{\mathrm{avg}}+\Delta k(\phi)$. This allows us to define a limb-darkened angular diameter $\left(\theta_{\mathrm{LD}}\right)$ through the relation $k_{\mathrm{avg}}=\frac{\theta_{\mathrm{UD}}}{\theta_{\mathrm{LD}}}$, which does not include the phase dependence of the limb darkening.

Assuming a distance of $d=275 \mathrm{pc}$ for $\delta$ Cep, we can determine the photospheric angular diameter $\theta_{\text {photo }}$ :

$\theta_{\text {photo }}[\mathrm{mas}]=9.305 \frac{R\left(\tau_{\mathrm{c}}=2 / 3\right)\left[R_{\odot}\right]}{d[\mathrm{pc}]}$

where $R\left(\tau_{\mathrm{c}}=2 / 3\right)$ is the photospheric radius corresponding to $\tau=2 / 3$ in the continuum, directly deduced from the model of $\delta$ Cep.

We can derive the uniform angular diameter $\theta_{\text {UD }}$ by applying a classical $\chi^{2}$ minimization algorithm between (i) the Hankel transform of the continuum-intensity distribution derived from the model and (ii) the Hankel transform of a uniform disk distribution with only one parameter, the angular diameter $\theta_{\mathrm{UD}}$, given by the following relationship:

$V\left(B_{\mathrm{p}}, \theta_{\mathrm{UD}}, \lambda\right)=\left|\frac{2 J_{1}(x)}{x}\right|$

where $x=15.23 \frac{B_{\mathrm{p}}[\mathrm{m}] \theta_{\mathrm{UD}}[\mathrm{mas}]}{\lambda[\mathrm{nm}]}$ is the spatial frequency, $J_{1}$ is the Bessel function of first order, and $B_{\mathrm{p}}$ the baseline of the interferometer projected on the sky.

Figure $3 \mathrm{a}$ shows $\theta_{\text {photo }}, \theta_{\mathrm{UD}}$, and $\theta_{\mathrm{LD}}$ against the pulsation phase. The $k$-factor, linked to the limb-darkening of the star, 

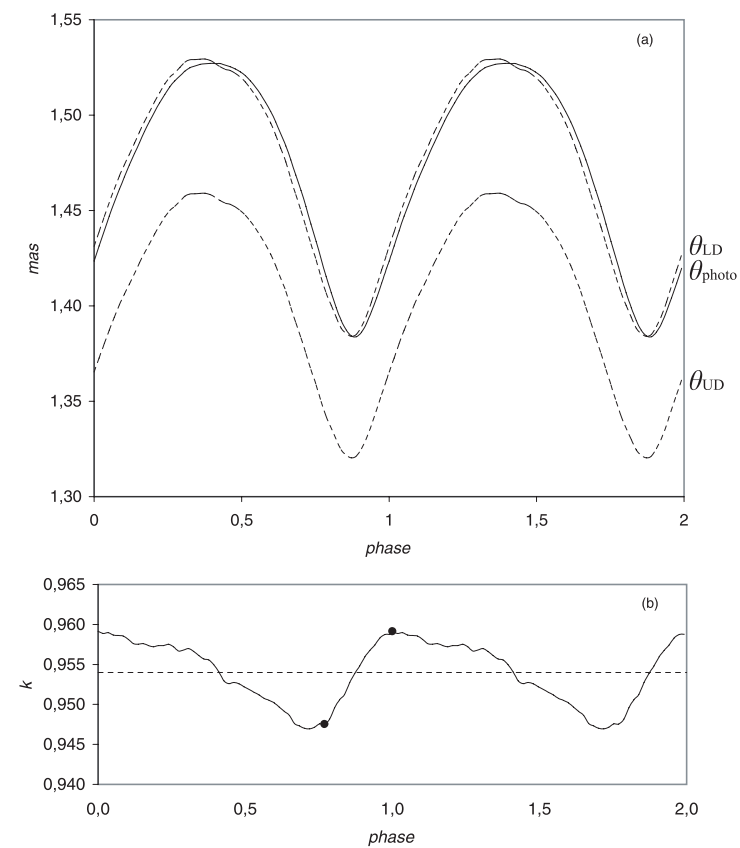

Fig. 3. a) Different theoretical angular diameters against phase. Dashed lines represent the uniform disk $\left(\theta_{\mathrm{UD}}\right)$ and limb-darkened $\left(\theta_{\mathrm{LD}}\right)$ angular diameters. The solid line is the photospheric angular diameter $\left(\theta_{\text {photo }}\right)$. b) The theoretical $k$ factor deduced from Eq. (1). The horizontal dashed line corresponds to $k_{\text {avg }}$. This $k$-factor is directly linked to the limb darkening of the star. The two dots correspond to the two considered phases.

is represented as a function of the pulsation phase in Fig. $3 b$. We find $k_{\text {avg }}=0.954$ and a peak to peak amplitude of 0.015 .

For the pulsation phase $\phi=0.77$, the profile is the most limb-darkened (see Fig. 2), so the $\theta_{\mathrm{UD}}$ and the $k$-factor are lower compared to the average value (see Fig. $3 \mathrm{~b}$ ). Also $\theta_{\mathrm{LD}}$ is lower compared to the reference angular diameter $\theta_{\text {photo }}$ (see Fig. 3a). The situation is the opposite at phase $\phi=0.0$.

Comparing $\theta_{\text {photo }}$ and $\theta_{\mathrm{LD}}$ directly shows the impact of the limb-darkening variation on the derived angular diameters. We find that the $\theta_{\mathrm{LD}}$ angular diameter curve is shifted by about 0.02 in phase compared to the photospheric angular diameter curve. Thus, considering a constant limb darkening can lead to a relative error on the angular diameter of no more than $1 \%$ in the visible band.

We now derive the distance by applying a classical minimization process between the photospheric radius $R\left(\tau_{\mathrm{c}}=2 / 3\right)$ and the $\theta_{\mathrm{LD}}$ using the following relation:

$d[\mathrm{pc}]=9.305\left(\frac{R_{\mathrm{photo}}(\phi)\left[R_{\odot}\right]}{\theta_{\mathrm{LD}}(\phi)[\mathrm{mas}]}\right)$.

We obtain $d=275 \mathrm{pc}$, the reference distance. Thus, considering $\theta_{\text {LD }}$ instead of $\theta_{\text {photo }}$ to derive the distance has no consequence. This means that the limb-darkening phase dependence does not affect the derived distance. Indeed, the distance is related to the inverse of the amplitude of the angular diameter curve. With the observed shift in phase, the amplitude is not affected (or marginally) and neither is the distance.

\section{Intensity distributions in the spectral line}

\subsection{Visibility versus the wavelength}

We first consider the Fe I $6003.012 \AA$ spectral line (Table 1) for the two cases $\phi=0.77$ and $\phi=0.0$. The synthetic profile is
Table 1. Spectral lines used in this study. Lines are given depending on their forming region: from the upper part to the lower part of the atmosphere. Line-forming regions have been determined using the hydrodynamical model defined by $\tau_{1}=2 / 3$ where $\tau_{1}$ is the optical depth at the centre of the line (see Fig. 8). The line depth (in percentage of the continuum) is indicated.

\begin{tabular}{lcc}
\hline \hline Name & Wavelength $(\AA)$ & $\begin{array}{c}\text { Line depth } \\
\text { (\% of the continuum) }\end{array}$ \\
\hline Fe I & 6003.012 & 18 \\
Fe I & 6056.005 & 16 \\
Fe I & 6380.743 & 9 \\
Ni I & 6378.247 & 5 \\
\hline
\end{tabular}

computed for $R=90000$. Results are represented in Figs. 4 and 5.

We can conclude that the limb darkening decreases (i.e. the limb becomes less dark) at wavelengths away from the rest wavelength. In other words, at maximum expansion (resp. contraction) velocity, the limb is less dark in the blue (resp. in the red) part of the spectral line profile.

We then perform a Hankel transform for each intensity distribution of Fig. 4 in the case of the maximum contraction velocity (Fig. 6a). Considering a typical projected baseline of $B_{\mathrm{p}}=65 \mathrm{~m}$, we derive the corresponding visibility for each line (Fig. 6b). The intensity distributions in the continuum (first and last points) lead to a visibility of about $V=0.485$. The effect on the visibility is thus about $13 \%$ (peak to peak) and looks asymmetric. In particular, the limb of the star is brighter for $\lambda=6002.63 \AA$ than its centre (Fig. 4), the star appears thus larger, and the corresponding visibility $(V=0.450)$ is lower than in the continuum (Fig. 6b). The relative effect on the visibility curve is $12 \%$, $8 \%$, and $5 \%$, considering spectral resolutions of $R=45000$, $R=23000$, and $R=12000$ (Fig. 7).

\subsection{Visibility versus the pulsation phase for four metallic lines}

The same procedure is performed for the six different pulsation phases indicated in Fig. 8a and for each considered spectral line of Table 1 , and the resolution is $R=90000$. The line-forming regions of the four spectral lines chosen for the study, as defined by $\tau_{1}=2 / 3$, are spread all over the atmosphere of the star. The corresponding mass zones or radii in the atmosphere of the star are represented in Fig. 8b. A zoom is given in Fig. 8c. From the deeper to upper parts of the atmosphere, we find the photosphere and the radii corresponding to lines: Ni I $6378.247 \AA$, Fe I $6380.743 \AA$, Fe I $6056.005 \AA$, and Fe I $6003.012 \AA$. As expected, line depths in percentage of the continuum (indicated in Table 1) are strongly correlated to the line forming regions.

The corresponding visibility curves are presented in Fig. 9 as a function of the velocity. For clarity, we have conserved the same angular resolution for the 4 spectral lines to obtain the same visibility level in the continuum.

\section{Discussion}

First, for a given spectral line, the amplitude of the signal in visibility (from peak to peak) is related to the absolute value of the photospheric pulsation velocity. In particular, when the pulsation velocity is zero, i.e. $\phi=0.39$ or $\phi=0.89$ (resp. maximum or minimum radius of the star) the visibility signature is marginal. However, we find that in the most favourable case $(\phi=0.77)$, 


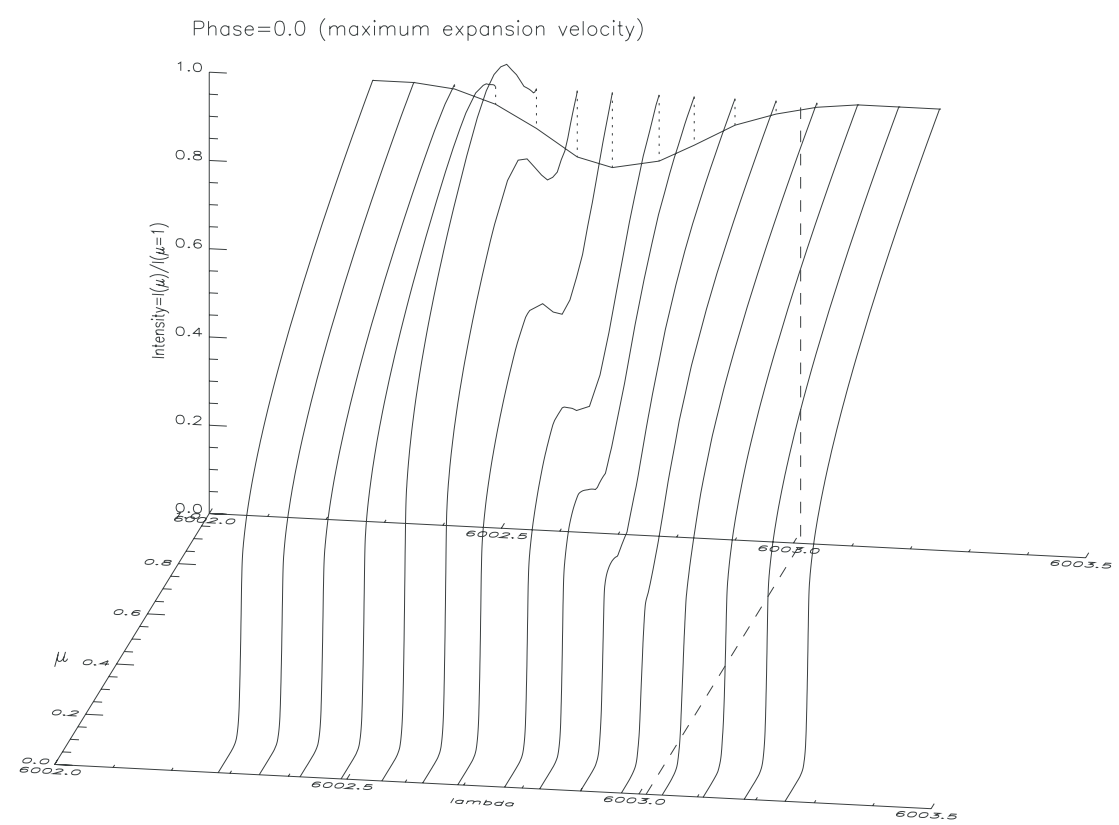

Fig. 4. A 3D diagram that represents the spectral line profile (Fe I 6003.012 $\AA$ ) for the maximum expansion velocity $(\phi=0.00)$ with the corresponding intensity distributions. The dashed line represents the reference wavelength in the stellar rest frame.

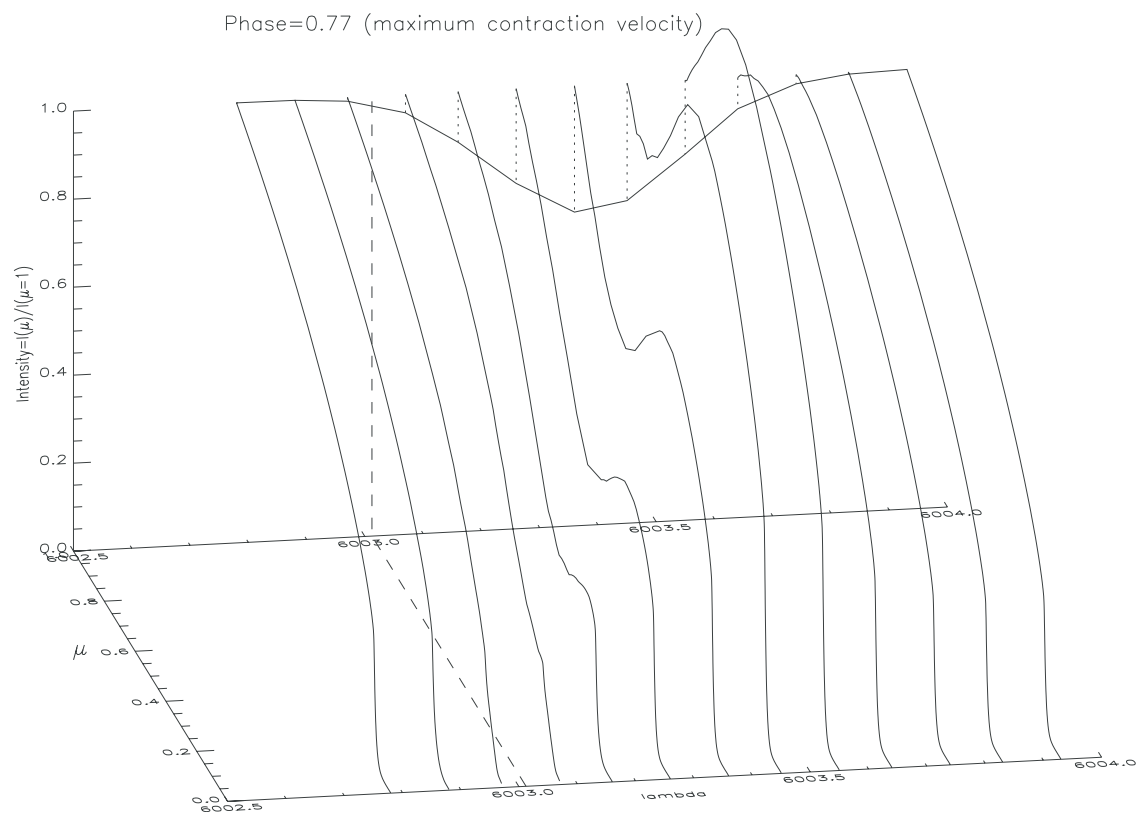

Fig. 5. Same as Fig. 4 but for the maximum contraction velocity $(\phi=0.77)$.

the visibility effect is about $19 \%$ for a resolution $R=90000$. For a resolution of $R=12000$, it corresponds to an effect of $7 \%$. This effect should be easily detected by spectro-interferometers when observing in the visible (see for example the VEGA project, Mourard et al. 2005). In the infrared, our model needs to be tested in detail, but a decrease in these effects of a few percent is expected. With the resolution $(R=12000)$ of the AMBER/VLTI instrument (Petrov et al. 2003), in $J, H, K$ bands these signatures in the visibility should be detectable for the maximum contraction or expansion velocity.

Second, for a given pulsation phase, the relative effect observed on the visibility curve between the four spectral lines is progressive and related to the line depth. Indeed, the intensity distribution signature is more important for a strong line than for a weak line (see for e.g. Figs. 4 and 5).
Third, the effect due to the line-forming region radius (see Figs. $8 \mathrm{~b}$ and c) is marginal. In fact, to constrain the projection factor we have to derive, for a given pulsation phase, the differential position of the spectral-lines forming regions or its equivalent, the velocity gradient in the atmosphere of the star. Nardetto et al. (2004) showed that measuring the radial velocity associated to the $\mathrm{Fe}$ I $6003.0123 \AA$ line induces a projection factor of $p=1.32$ if one wants to obtain the radius corresponding to the line forming region after integration. Conversely, in the case of the IBW method, the value needed to reach the photospheric radius (corresponding to $\tau=2 / 3$ in the continuum) should be $p=1.27$. Thus, due to velocity gradient in the $\delta$ Cep atmosphere, we have an average difference between the two projection factors of about $4 \%$. This difference is directly linked to a similar average difference of $4 \%$ between the variation in the photospheric 

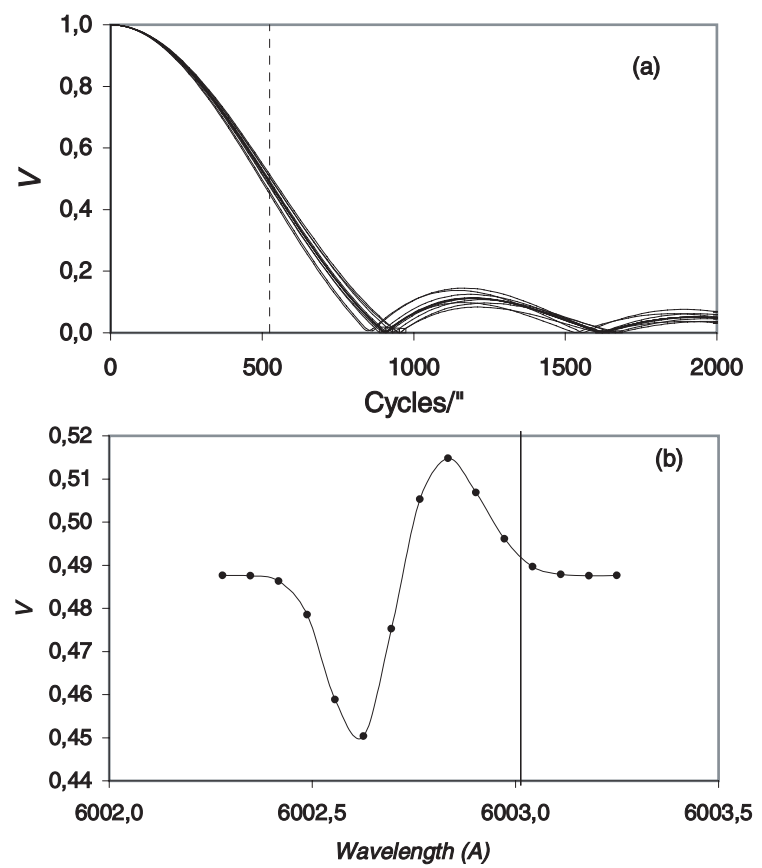

Fig. 6. a) The Hankel transform of each intensity distribution of Fig. 4 in cycle/". The vertical dashed line corresponds to the resolution obtained with a baseline of 65 meters. b) Visibility as a function of the wavelength.
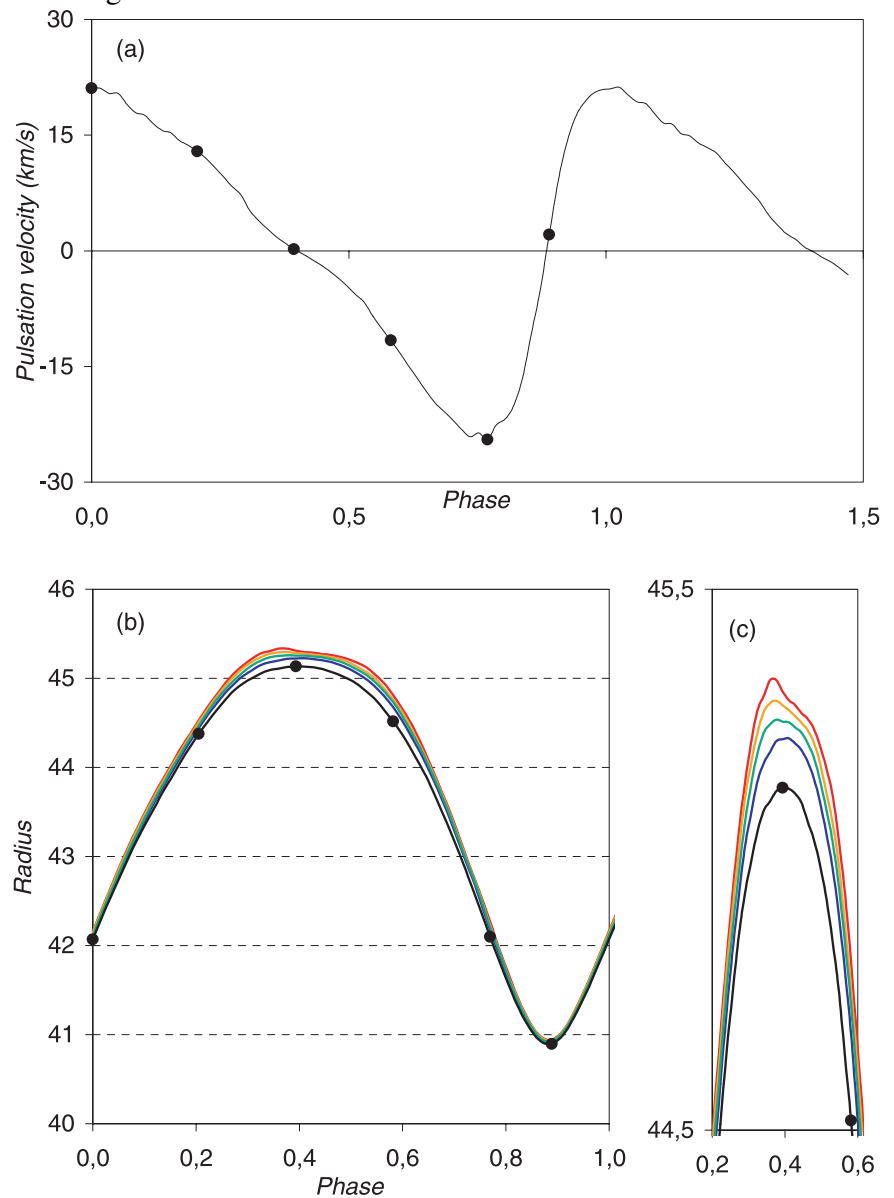

Fig. 8. a) The pulsation velocity corresponding to the photosphere is represented against the pulsation phase (in the stellar rest frame). Six different phases (black points) are considered in this study. b) The photospheric radius, defined as $\tau_{\mathrm{c}}=2 / 3$, and the line-forming region radius, defined as $\tau_{1}=2 / 3$, are represented as functions of the pulsation phase. We have (down to up) the photospheric radius and the radii corresponding to the lines Ni I 6378.247 A, Fe I $6380.743 \AA$ A , Fe I $6056.005 \AA$, and Fe I $6003.012 \AA$. c) The same as b) but enlarged around the phase of the maximum radius of the star.

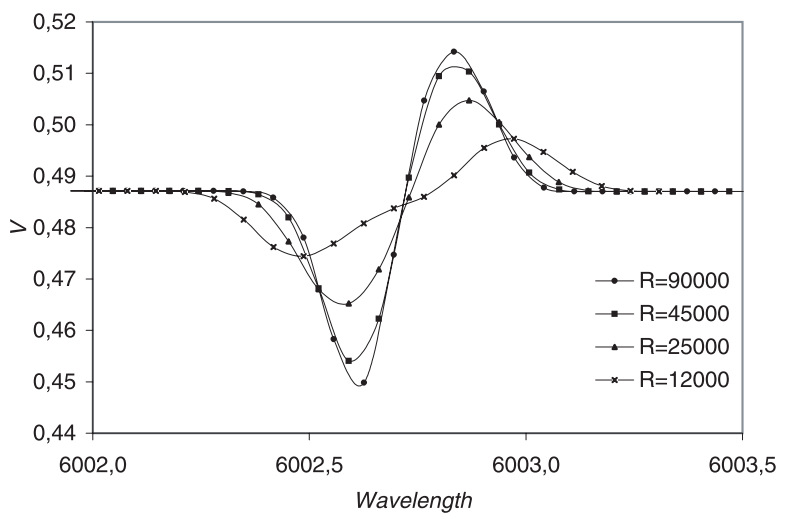

Fig. 7. Visibility as a function of wavelength for a projected baseline of 65 meters and for different spectroscopic resolutions.

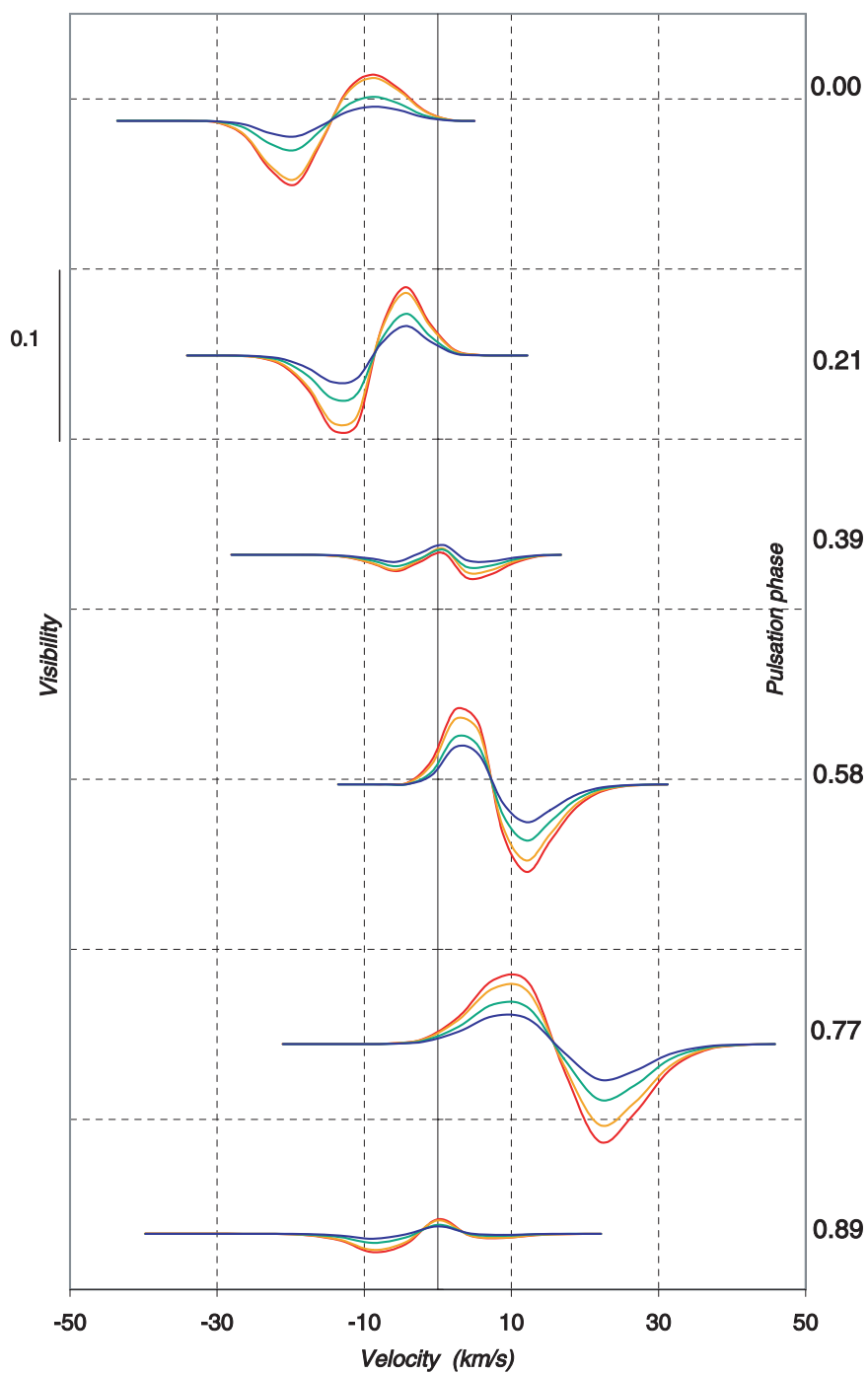

Fig. 9. The visibility is represented as a function of the velocity in the case of the four spectral lines and for each pulsation phase. The resolution is $R=90000$. The pulsation phase is indicated on the right side of the diagram. The visibility curves are in the same order as the radius curves (or line depth), spectral lines forming in the upper part of the atmosphere (strong lines) lead to an important effect on the visibility curve while lines forming in the lower part (weak lines), near the photosphere, lead to a minor effect on the visibility. There is a direct correspondence between the line depth and the visibility signature. The effect due to the line-forming region radius is marginal. 
and line-forming region radius (see Fig. $8 \mathrm{~b}$ and c). Such an effect on the amplitude of the visibility signatures is present but dominated by the line-depth effect. As already mentioned, we obtain for the maximum pulsation velocity an effect of $19 \%$ on the visibility curve (in the visible). Considering a $4 \%$ difference due to velocity gradient, we find an effect of $1 \%$, which is marginally detectable by spectro-interferometers.

Finally, constraining the projection factor by using only the visibility signature seems difficult. This is an important conclusion of this work. However, it should be possible to reach such a goal by using different observables, together with the visibility signature, such as the spectral line profile (Nardetto et al. 2005) and the differential interferometry (i.e. the photocentre displacement). Note that a precision of $1 \mu$ as can be reached with AMBER/VLTI $\left(\lambda=1 \mu \mathrm{m}\right.$ and $\left.B_{\mathrm{p}}=200 \mathrm{~m}\right)$ in differential interferometry (Mourard \& Nardetto 2005). To prepare such observations, we are currently including the rotation velocity in the radiative transfert of our hydrodynamic model and also testing the model in the infrared.

\section{Conclusion}

We used a hydrodynamical model of $\delta$ Cep to derive intensity distributions in the continuum and in the four spectral lines for which the model predicts that they form gradually in different regions of the atmosphere of the star Fe I $6003.012 \AA$, Fe I $6056.005 \AA$, Fe I $6380.743 \AA$, and Ni I $6378.247 \AA$.

Intensity distributions in the continuum have been used to study the limb darkening of the star. We find a peak-to-peak variation of the $k$-factor curve of 0.015 . This leads to a systematic shift in phase on the derived angular diameter curve of 0.02 . However, the derived distance is not affected, because it is linked to the amplitude of the angular diameter curve, which is only slightly changed by the shift effect. Thus, considering the time-dependence of the limb-darkening doest not seem to be a priority in the context of the IBW method. In near future, long-baseline interferometers will, however, be able to measure the limb-darkening directly against the pulsation phase. It will be possible to constrain such pulsating star modelling and thus bring new insights in atmospheric dynamics.

Intensity distributions in the spectral lines were used to derive the visibility as a function of wavelength for differentpulsation phases. We find an effect on the visibility in most favourable cases (i.e. in the visible and at the maximum pulsation velocity phase) to about $19 \%$ for a spectroscopic resolution of $R=90000$ and $7 \%$ for $R=12000$. Such effects should be detected byspectro-interferometers (in the visible with the VEGA project or in the infrared with AMBER/VLTI instrument).

We note a correspondence between the line depth and the amplitude of the visibility signature. The impact of the line forming region radius on the visibility (important to study velocity gradient and the projection factor) seems to be marginal (about 1\%). However, the wavelength- and phase-dependent visibility curves can be used, together with the spectral line profiles and the photocenter displacement (differential interferometry), to constrain Cepheids' physical parameters.

\section{References}

Breitfellner, M. G., \& Gillet, D. 1993, A\&A, 277, 524

Fokin, A. B., Gillet, D., \& Breitfellner, M. G. 1996, A\&A, 307, 503

Kervella, P., Nardetto, N., Bersier, D., et al. 2004a, A\&A, 416, 941

Marengo, M., Sasselov, D. D., Karovska, M., et al. 2002, ApJ, 567, 1131

Marengo, M., Karovska, M., Sasselov, D. D., et al. 2003, ApJ, 589, 975

Marengo, M., Karovska, M., Sasselov, D. D., et al. 2004, ApJ, 603, 285

Mérand, A., Kervella, P., Coude du Foresto, V., et al. 2005, A\&A, 438, L9

Mourard, D., \& Nardetto, N. 2005, Col. Stellar Pulsation And Evolution, Rome, in press

Mourard, D., Antonelli, P., et al. 2005, ESO Conf., Garching, in press

Nardetto, N., Fokin, A., Mourard, D., et al. 2004, A\&A, 428, 131

Nardetto, N., Mourard, D., Kervella, P., et al. 2005, A\&A, submitted

Petrov, R. G., Malbet, F., Weigelt, G., et al. 2003, in Interferometry for Optical Astronomy II, ed. W. A. Traub., 4838 (SPIE), 924

Sasselov, D. D., \& Karovska, M. 1994, 432, 367 